Relationship between the optical absorption and the density of deep gap states in microcrystalline silicon

Stefan Klein, Friedhelm Finger, Reinhard Carius, Thorsten Dylla, and Josef Klomfass

Citation: Journal of Applied Physics 102, 103501 (2007);

View online: https://doi.org/10.1063/1.2815645

View Table of Contents: http://aip.scitation.org/toc/jap/102/10

Published by the American Institute of Physics

Articles you may be interested in

Hydrogen in amorphous and microcrystalline silicon films prepared by hydrogen dilution Journal of Applied Physics 80, 4971 (1998); 10.1063/1.363541

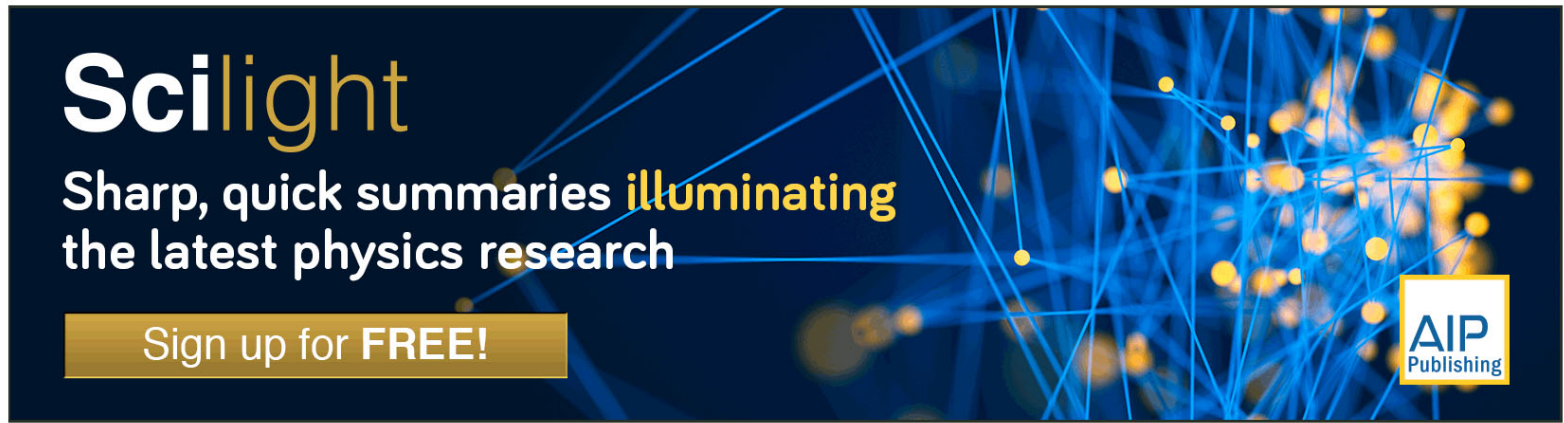




\title{
Relationship between the optical absorption and the density of deep gap states in microcrystalline silicon
}

\author{
Stefan Klein, ${ }^{\text {a) }}$ Friedhelm Finger, ${ }^{\text {b) }}$ Reinhard Carius, Thorsten Dylla, and Josef Klomfass \\ Institut für Energieforschung-Photovoltaik, Forschungszentrum Jülich, 52425 Jülich, Germany
}

(Received 27 June 2007; accepted 26 September 2007; published online 19 November 2007)

\begin{abstract}
We have measured the subgap optical absorption of undoped microcrystalline silicon samples with photothermal deflection spectroscopy and compared it with the spin density measured by electron spin resonance. The material was prepared using the hot wire chemical vapor deposition technique with a broad range of deposition parameters, yielding materials with a wide variety of structural, optical, and electronic properties. A nearly linear correlation between the spin densities and the subgap absorption coefficient at photon energy of $0.7 \mathrm{eV}$ over three orders of magnitude indicates that the optical absorption in this region is due to the dominating paramagnetic defects, likely dangling bonds. However, the structural composition of the material, determined by Raman spectroscopy, has also some influence on the optical and electronic properties, which leads to some deviation from a straightforward relationship between optical absorption and spin or defect density. (c) 2007 American Institute of Physics. [DOI: 10.1063/1.2815645]
\end{abstract}

\section{INTRODUCTION}

The obvious structural defect in amorphous and microcrystalline silicon $(a-\mathrm{Si}: \mathrm{H}$ and $\mu \mathrm{c}-\mathrm{Si}: \mathrm{H})$ is the dangling bond. It is widely accepted that dangling bonds are the cause for the dominating electronic defect in the mobility gap in $a-\mathrm{Si}: \mathrm{H}$ and this defect is to a large extent responsible for the charge carrier properties via recombination and trapping ${ }^{1}$. The dangling bonds in $a$-Si: $\mathrm{H}$ are identified by electron spin resonance (ESR) measurements which detect the dangling bond in a singly occupied, neutral charge state. In $\mu c-\mathrm{Si}: \mathrm{H}$ the situation is more complicated as the dangling bond type defect could be located in different local environments of the material, crystalline, and amorphous regions and grain boundaries. Nevertheless also in $\mu \mathrm{c}-\mathrm{Si}: \mathrm{H}$ there is a good correlation between spin density and the material quality, which has been successfully used for material and device development. ${ }^{2}$ But measurement of these dangling bonds in $a-\mathrm{Si}: \mathrm{H}$ and $\mu \mathrm{c}-\mathrm{Si}: \mathrm{H}$ by electron spin resonance can be laborious and afflicted with some uncertainty. Alternative, easier to use methods for determination of the defect density are therefore looked for. For $a-\mathrm{Si}: \mathrm{H}$ this was achieved by finding a linear correlation of the spin density with the optical absorption coefficient $\alpha$ in the subgap region. ${ }^{3,4}$ Thus, the measurement of $\alpha$ provides an easier method to access the material quality. In particular, photothermal deflection spectroscopy (PDS) with its dynamical range of more than five orders of magnitude of absorption coefficient is suitable for such measurement. ${ }^{3}$ PDS detects optical transitions between all defect states independently from the transport properties which is an advantage over photoconductivity spectroscopy, which is also frequently used to measure optical absorption ${ }^{5,6}$ A similar correlation between below-band gap

\footnotetext{
${ }^{a)}$ Present address: Applied Materials, Siemensstr. 100, 63755 Alzenau, Germany.

b) Author to whom correspondence should be addressed. Electronic mail: f.finger@fz-juelich.de
}

optical absorption and defect density was observed for polycrystalline silicon. ${ }^{7}$ For the to date intensively investigated $\mu \mathrm{c}-\mathrm{Si}: \mathrm{H}$ such a relation was proposed, ${ }^{8-11}$ but has yet not been shown convincingly by systematic experimental data. The heterogeneous structure of $\mu \mathrm{c}-\mathrm{Si}: \mathrm{H}$ might be one reason for the difficulty in finding a similar correlation there. $\mu \mathrm{c}-\mathrm{Si}: \mathrm{H}$ is a composition of amorphous and crystalline regions with grain boundaries and voids. The crystalline regions consist of columnar grain clusters which are built up from small crystallites. ${ }^{12,13}$ The size of the crystallites and the columns depends on the deposition conditions. The structural composition affects the optical and electronic properties of the material. A typical optical absorption spectrum of $\mu \mathrm{c}-\mathrm{Si}: \mathrm{H}$ is shown together with the spectra of $a-\mathrm{Si}: \mathrm{H}$ and $c$-Si in Fig. 1(a). Between photon energy of 1.1 and $1.6 \mathrm{eV}$ the optical absorption of $\mu \mathrm{c}-\mathrm{Si}: \mathrm{H}$ is similar to crystalline silicon. At higher photon energies $(E>1.7 \mathrm{eV})$ amorphous material fractions lead to an enhanced absorption, scattering at the surface and at grain boundaries can also increase the absorption. ${ }^{14}$ At low photon energies near the crystalline band edge, the absorption is enhanced due to band tail states and defects like, e.g., dangling bonds lead to absorption below the band gap. It is this region which is the matter of concern here. However, it can be expected that the contributions of defect or tail state absorption of the various structure phases overlap in a yet unknown way. Therefore, whether a simple relationship between this subband gap absorption and defect density in $\mu \mathrm{c}-\mathrm{Si}: \mathrm{H}$ exists and whether these defects are relevant for the charge carrier transport properties needs experimental verification, in particular in view of the wide range of structure compositions of the $\mu \mathrm{c}-\mathrm{Si}: \mathrm{H}$ material.

In Figs. 1(b) and 1(c) ESR spectra of undoped $a-\mathrm{Si}: \mathrm{H}$ and $\mu \mathrm{c}-\mathrm{Si}: \mathrm{H}$ are shown. The $a-\mathrm{Si}: \mathrm{H}$ signal is an almost symmetric line with a Gaussian-type line shape. $\mu \mathrm{c}-\mathrm{Si}: \mathrm{H}$ frequently shows a more pronounced structure. The $g$ value of the resonance center in both materials is slightly but distinctly different. Both spectra are taken on powder samples. 

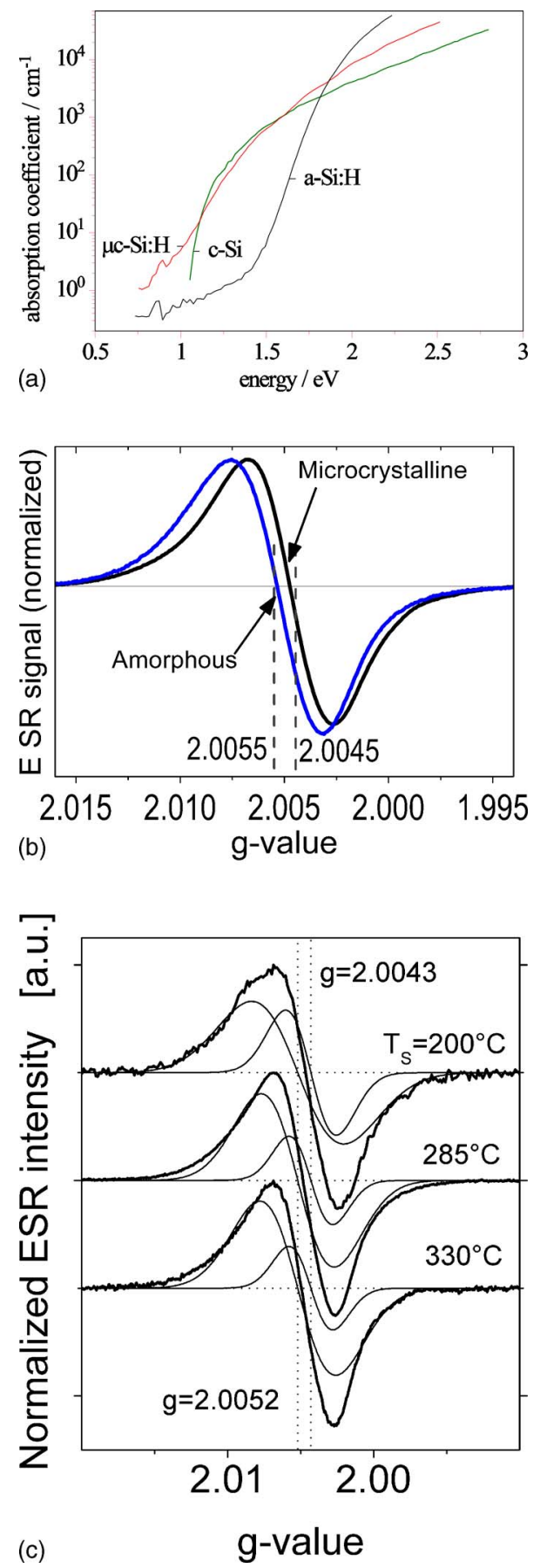

FIG. 1. (Color online) (a) Optical absorption coefficient of $c-\mathrm{Si}, \mu \mathrm{c}-\mathrm{Si}: \mathrm{H}$, and $a-\mathrm{Si}: \mathrm{H}$ vs photon energy; (b) comparison of ESR spectra of $a-\mathrm{Si}: \mathrm{H}$ and $\mu \mathrm{c}-\mathrm{Si}: \mathrm{H}$; and (c) ESR spectra of $\mu \mathrm{c}-\mathrm{Si}: \mathrm{H}$ with high crystalline volume fraction and (in gray) two Gaussian lines used for deconvolution.

The signals appear as if arising from isotropic defects. A strong defect- or better $g$-tensor anisotropy would result in a pronounced powder pattern for the powder samples. For amorphous silicon this is not observed. It is argued that the powder pattern is overlapping with inhomogeneous broadening due to structural disorder which results in a more or less isotropic signal. ${ }^{15}$ For $\mu \mathrm{c}-\mathrm{Si}: \mathrm{H}$ one can describe the ESR signal as a superposition of several, at least two, resonances due to dangling bond type defects in different environments. ${ }^{16-18}$ The identification and location of the possible different defects is still a matter of debate. See ex- amples of spectra deconvolution in Fig. 1(c). But the distinct signal feature has been interpreted also in terms of an anisotropic defect. ${ }^{19,20}$ For our study we shall not distinguish between these different interpretations and we will calculate the spin density from an integration over the entire signal in $\mu \mathrm{c}-\mathrm{Si}: \mathrm{H}$.

We present and discuss the results of a systematic comparison of previously published data ${ }^{21,22}$ of the spin density and subgap absorption of $\mu \mathrm{c}-\mathrm{Si}: \mathrm{H}$ for a variety of $\mu \mathrm{c}-\mathrm{Si}: \mathrm{H}$ samples with a different crystalline volume fraction prepared by hot wire chemical vapor deposition (CVD) ${ }^{21,22}$ In all cases the optical and the spin data are taken on codeposited material. As a conclusion, we propose that subband gap optical absorption detected with photothermal deflection spectroscopy can be used to evaluate the defect density in $\mu \mathrm{c}-\mathrm{Si}: \mathrm{H}$ with appropriate caution.

\section{EXPERIMENT}

The samples used for our measurements were thin films of $\mu \mathrm{c}-\mathrm{Si}: \mathrm{H}$ prepared by hot wire chemical vapor deposition (HWCVD) using a tantalum wire arrangement. ${ }^{21,22}$ The films were deposited from a silane/hydrogen mixture with various silane concentrations $\mathrm{SC}=\left[\mathrm{SiH}_{4}\right] /\left[\mathrm{SiH}_{4}+\mathrm{H}_{2}\right]$ at filament temperatures of $T_{F}=1650-1800{ }^{\circ} \mathrm{C} .2-3 \mu \mathrm{m}$ thick films were prepared on glass substrates for PDS measurements and on aluminum foil for the ESR samples. The powder used in ESR measurements was obtained from the $\mathrm{Al}$ foil by a $\mathrm{HCl}$ etch and sealed in He atmosphere after they were rinsed in de-ionized water and dried at ambient. The ESR spectra were taken in the $X$ band at room temperature; spin densities were derived from a numerical integration of the experimental spectra and compared with a spin standard. ${ }^{23}$

PDS measurements were performed at room temperature using $\mathrm{CCl}_{4}$ as the deflection medium. Monochromatic light from a halogen and xenon lamp was used for illumination; the deflection was measured with a semiconductor laser in lock-in technique. The transmission of the sample was measured simultaneously. Interference fringes due to multiple reflections in optically homogeneous films can be removed by a modified evaluation procedure based on the Ritter-Weiser formula. ${ }^{24}$ However, fringes that are modified due to inhomogeneities of the absorption coefficient in the growth direction cannot be removed. ${ }^{25}$ In addition, the contributions of the substrate absorption which overlap with the below-band gap absorption of the $\mu \mathrm{c}-\mathrm{Si}: \mathrm{H}$ can be separated using the phase shift between probe beam and signal. Raman spectra were measured directly on the Al-foil samples with an excitation wavelength of $647 \mathrm{~nm}$. The integrated intensity ratio $I_{C}^{\mathrm{RS}}=I_{C} /\left(I_{A}+I_{C}\right)$ was determined by deconvoluting the spectra into two Gaussian peaks at 500 and $520 \mathrm{~cm}^{-1}$, attributed to the crystalline phase and a Gaussian peak at $480 \mathrm{~cm}^{-1}$, attributed to the amorphous phase. ${ }^{12}$ All samples have an intrinsic character, as indicated by low dark conductivities of $2 \times 10^{-6} \mathrm{~S} / \mathrm{cm}$ and below. ${ }^{21,22}$

\section{RESULTS}

Figure 2(a) shows the optical absorption spectra of $\mu \mathrm{c}-\mathrm{Si}: \mathrm{H}$ films prepared with different $\mathrm{SC}$ at $T_{S}=250{ }^{\circ} \mathrm{C}$. 

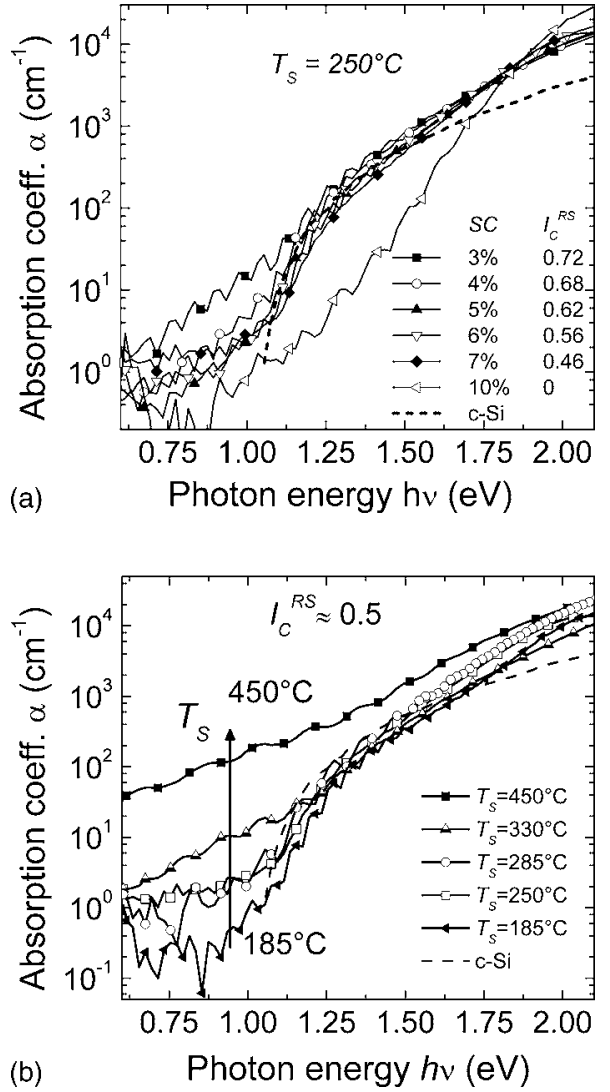

FIG. 2. Optical absorption spectra of $\mu \mathrm{c}-\mathrm{Si}: \mathrm{H}$ samples measured by PDS. (a) Films deposited with different silane concentration SC and the indicated $I_{C}^{\mathrm{RS}}$ values. (b) Films prepared near the $\mu \mathrm{c}-\mathrm{Si}: \mathrm{H} / a-\mathrm{Si}: \mathrm{H}$ transition $\left(I_{C}^{\mathrm{RS}}\right.$ $\approx 0.5$ ) at different substrate temperatures $T_{S}$.

The variation of $\mathrm{SC}$ results in $\mu \mathrm{c}-\mathrm{Si}: \mathrm{H}$ films with various structural composition covering the entire range from highly crystalline $\left(I_{C}^{\mathrm{RS}}>0.7\right)$ to predominantly amorphous material $\left(I_{C}^{\mathrm{RS}}=0\right)$. The optical absorption follows the behavior as described in Fig. 1 and in the literature. ${ }^{8,14,26}$ At photon energies smaller than $1.1 \mathrm{eV}$, states within the band gap, e.g., dangling bonds, tail states, or other deep defects, contribute to the optical absorption of the films. With decreasing crystallinity, the absorption in the high energy region systematically increases due to the higher amorphous fraction. At the same time the absorption in the region between 1.1 and 1.7 $\mathrm{eV}$ decreases which is the energy range where the crystalline silicon absorption dominates. ${ }^{22}$ Below the crystalline band gap of $1.1 \mathrm{eV}$, a systematically decreasing absorption with decreasing photon energy is observed. For most of the samples this subgap absorption can be well approximated by an exponential behavior.

The effect of the deposition temperature in the HWCVD process on the optical absorption in $\mu \mathrm{c}-\mathrm{Si}: \mathrm{H}$ is shown in Fig. 2(b). The samples are $\mu \mathrm{c}-\mathrm{Si}: \mathrm{H}$ films with a crystalline volume fraction $I_{C}^{\mathrm{RS}} \sim 0.5$ prepared at different $T_{S}$ in the range between 185 and $450{ }^{\circ} \mathrm{C}$. The absorption in the subgap region increases continuously by almost three orders of magnitude as a function of substrate temperature. At higher energies $(>1.6 \mathrm{eV})$ some nonsystematic variation of the absorption is observed.

Figure 3 shows exemplarily the ESR spectra of material

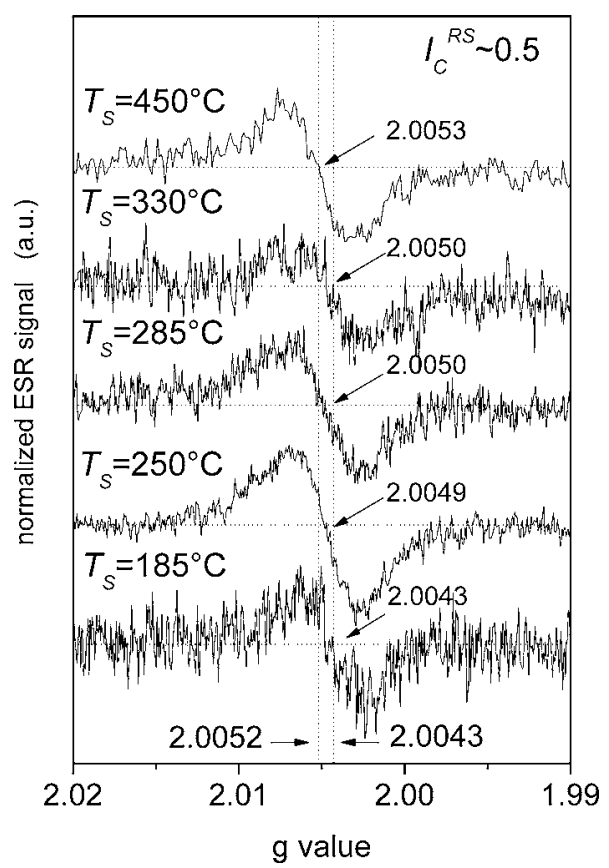

FIG. 3. ESR spectra normalized to the same peak-to-peak height of the samples in Fig. 2(b).

prepared in the same run as the films used for the PDS measurement, which are plotted in Fig. 2(b). All spectra show the typical asymmetric line shape with contributions at $g$ $=2.0043$ and $g=2.0052$. Also indicated in Fig. 3 are the $g$ values of the individual spectra, defined as the zero intensity crossing of the ESR signal.

In Fig. 4 the spin densities $N_{S}$ of a large number of samples prepared with different substrate temperatures $T_{S}$ versus the Raman intensity ratio $I_{C}^{\mathrm{RS}}$ are shown. Within the predominantly crystalline regime $\left(I_{C}^{\mathrm{RS}}>0.4\right)$ the spin density decreases with increasing amorphous phase content and increases again for material dominated by amorphous phase content $\left(I_{C}^{\mathrm{RS}}<0.4\right)$. Only for the highest $T_{S}$ of $450{ }^{\circ} \mathrm{C} N_{S}$ decreases monotonously, however, at very high values of $N_{S}>10^{17} \mathrm{~cm}^{-3}$. A systematic decrease of $N_{S}$ with decreasing $T_{S}$ can be observed independently of the particular structure composition; $N_{S}$ decreases by almost three orders of magnitude to very low values of $N_{S}=4 \times 10^{15} \mathrm{~cm}^{-3}$ observed for

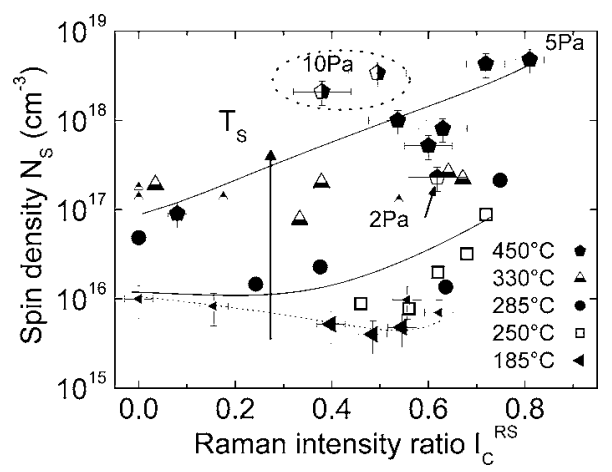

FIG. 4. Spin density $N_{S}$ vs Raman intensity ratio $I_{C}^{\mathrm{RS}}$ for samples prepared at different $T_{S}$ with various SC. Small symbols indicate samples which pealed off before PDS measurement. At $T_{S}=450{ }^{\circ} \mathrm{C}$ different $p_{D}$ were applied during deposition. 


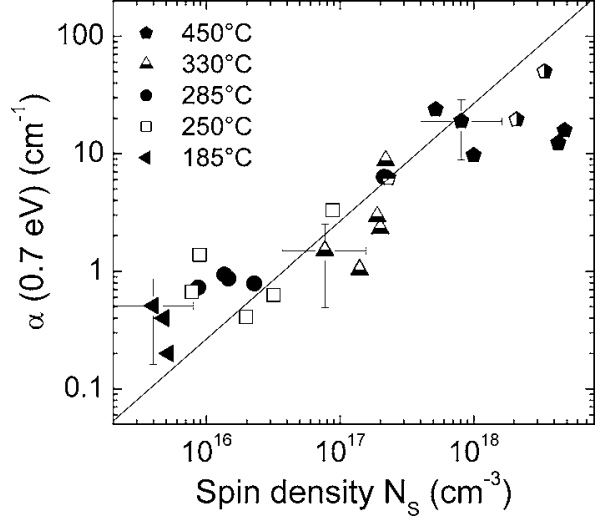

FIG. 5. Subgap absorption $\alpha_{0.7 \mathrm{eV}}$ vs total spin density $N_{S}$. The line indicates a linear fit to the data. See also Fig. 4 for symbols.

material prepared at $T_{S}=185{ }^{\circ} \mathrm{C}$ and $I_{C}^{\mathrm{RS}} \sim 0.4$. Such material, incorporated in solar cells yields the highest conversion efficiencies of up to $\eta=9.4 \% .^{21}$ For $T_{S}=450{ }^{\circ} \mathrm{C}$ and medium crystallinity, films were also prepared with different deposition pressures $p_{D}$ between 2 and $10 \mathrm{~Pa}$, leading to an increase of the $N_{S}$ by more than one order of magnitude. ${ }^{21}$ Comparing Figs. 2(b) and 4 some systematic similarities can be observed. Increasing the substrate temperature from 185 to $450{ }^{\circ} \mathrm{C}$ not only results in an increase of both the subgap absorption and $N_{S}$, also the magnitude of the effects are the same. With a similar behavior reported for $a-\mathrm{Si}: \mathrm{H}$ and poly-Si (Refs. 3 and 7) it is tempting to correlate these two quantities also for $\mu \mathrm{c}-\mathrm{Si}: \mathrm{H}$. The optical absorption within the subgap region, averaged between 0.65 and $0.75 \mathrm{eV}$, is shown in Fig. 5 as a function of the spin density $N_{S}$ for $\mu \mathrm{c}-\mathrm{Si}: \mathrm{H}$ for various structure compositions. To see in how far there is a linear relationship between $\alpha_{0.7 \mathrm{eV}}$ and $N_{S}$ similar to the relationship between defect absorption and spin density in $a$-Si: $\mathrm{H}$, we perform a linear fit which is shown as the solid line in Fig. 5. It approximates the experimental data fairly well over three orders of magnitude of $N_{S}$. For the relationship $N_{S}=A \alpha_{0.7 \mathrm{eV}}\left(\mathrm{cm}^{-1}\right)$ a proportionality constant of $A=3.6(5) \times 10^{16} \mathrm{~cm}^{-2}$ is obtained between the spin density and the optical absorption coefficient. Possible reasons for the deviation from the linear behavior shall be discussed later.

\section{DISCUSSION}

From Fig. 5 we propose an almost linear correlation between the spin density and the optical absorption below the band edge. This dependency of $\alpha_{0.7 \mathrm{ev}}$ on $N_{S}$ over three orders of magnitude suggest that the dominating paramagnetic defect, most likely the dangling bond, is the main state contributing to the subband gap absorption. The proportionality constant of $A=3.6(5) \times 10^{16} \mathrm{~cm}^{-2}$ is similar to the values previously obtained for amorphous or polycrystalline silicon. $^{3,4,7}$

However, there are comparatively small, but systematic deviations from a linear behavior: On the one hand, for the highest and lowest spin densities, and on the other hand, for the different structural compositions within the individual temperature series. Various reasons, some related to the limi- tations of the experiments, others due to the physical nature of the material, account for these deviations. The sample preparation procedure of the ESR samples, i.e., the etching with $\mathrm{HCl}$ and rinsing in water, can result in a considerable increase of the spin density of up to a factor of 3 in highly crystalline samples. ${ }^{17,27}$ The samples for the optical absorption measurements were not exposed to such a treatment. The measured spin densities of the highly crystalline samples might therefore be higher than in the corresponding PDS samples. How far the optical absorption is affected, e.g., by storage in air as well, is not known. Anyway for medium range crystalline volume fractions no or only minor influence of the ESR powder samples treatment on the spin density was observed. $^{17,27}$

For the low optical absorptions in the subgap region, contributions from the substrate to the signal cannot be excluded despite the applied phase correction of the signal which allows a partial removal of the substrate contribution. $^{24}$ This might lead, together with the contribution of interface defects, to an offset of the absorption coefficient at low $N_{S}$.

Furthermore, shifts of the Fermi level due to charging effects at the grain boundaries by adsorbed gases like water vapor or oxygen can change the occupation of midgap states in the material. ${ }^{27}$ For materials with low dangling bond density, already small amounts of charge are sufficient to significantly shift the Fermi level and charge the dangling bonds into $D^{+}$or $D^{-}$states which are not detectable in ESR. In defect rich material, on the other hand, a much higher concentration of adsorbed donors or acceptors is needed in order to shift the Fermi level by a relevant amount to charge the dangling bonds.

Finally, the structural composition of the material will affect the optical absorption and the dangling bond density. Variations of the crystallinity come along with changes of the crystallite size, microstructure, and morphology of the samples. ${ }^{12}$ An enhanced optical absorption due to internal and surface scattering was observed for microcrystalline silicon. $^{8,9,26}$ The surface roughness and the distribution of crystalline regions depend on the deposition conditions and the crystallinity of the films. For the material prepared at high substrate temperatures, it is known that they have a pronounced amorphous nucleation layer even at high crystallinity. ${ }^{21,22,28,29}$ The optical cross section of a dangling bond in amorphous silicon at a photon energy of $0.7 \mathrm{eV}$ is expected to be much lower than in crystalline silicon due to the lower density of final states. Even if such $a-\mathrm{Si}: \mathrm{H}$ material has a high spin density, this will result in a lower corresponding subgap absorption at this photon energy.

Additionally, the density of states near the crystalline band gap might be lower in material with low crystalline fraction and the absorption coefficient is thus reduced. Photoluminescence investigations also show an increase of the tail state density in highly crystalline material ${ }^{30}$ which extend deep into the band gap and consequently contribute to the absorption. Differences of the transition matrix element in amorphous and microcrystalline silicon might cause further deviations. The location of most dangling bonds in the grain 
boundary region ${ }^{2}$ might lead to similar effects because the effective density of states there might be significantly lower than in crystalline regions.

However, despite all these problems, a surprisingly good linear correlation between the spin density and the optical absorption below the optical band gap is found, suggesting that the dangling bond is in fact the main contributor to the absorption at low energy.

\section{CONCLUSIONS}

In microcrystalline silicon the subband gap absorption measured by photothermal deflection spectroscopy and the spin density measured by electron spin resonance show a nearly linear relationship over three orders of magnitude with a proportionality constant of $A=3.6(5) \times 10^{16} \mathrm{~cm}^{-2}$ at $0.7 \mathrm{eV}$ photon energy. This relationship is affected by the heterogeneous nature of the $\mu \mathrm{c}-\mathrm{Si}: \mathrm{H}$ structure composition and the resulting influence on the measured and evaluated absorption and spin density values. The height of the optical subband gap absorption can be used as an optimization tool for preparation processes, however, a fine tuning for low absorption or spin density values might be difficult.

\section{ACKNOWLEDGMENTS}

We thank M. Hülsbeck for Raman measurements and S. Reynolds for helpful discussions.

${ }^{1}$ R. A. Street, Hydrogenated Amorphous Silicon (Cambridge University Press, Cambridge, 1991).

${ }^{2}$ F. Finger, L. Baia Neto, R. Carius, T. Dylla, and S. Klein, Phys. Status Solidi C 1, 1248 (2004).

${ }^{3}$ W. B. Jackson and N. M. Amer, Phys. Rev. B 25, 5559 (1982).

${ }^{4}$ N. Wyrsch, F. Finger, T. J. McMahon, and M. Vanecek, J. Non-Cryst. Solids 137-138, 347 (1991).

${ }^{5}$ M. Vanecek, J. Kocka, J. Stuchlik, Z. Kozizek, O. Stika, and A. Triska, Sol. Energy Mater. 8, 411 (1983).

${ }^{6}$ M. Günes, O. Göktas, S. Okur, N. Isik, R. Carius, J. Klomfass, F. Finger, and J. Optoelectron, Adv. Mater. 7, 161 (2005).

${ }^{7}$ W. B. Jackson, N. M. Johnson, and D. K. Biegelsen, Appl. Phys. Lett. 43, 195 (1983).

${ }^{8}$ M. Vanecek, A. Poruba, Z. Remes, J. Rosa, S. Kamba, V. Vorlicek, J. Meier, and A. Shah, J. Non-Cryst. Solids 266-269, 519 (2000).
${ }^{9}$ N. Beck, J. Meier, J. Fric, Z. Remes, A. Poruba, R. Fluckiger, J. Pohl, A. Shah, and M. Vanecek, J. Non-Cryst. Solids 198-200, 903 (1996).

${ }^{10}$ W. Bronner, R. Brüggemann, and M. Mehring, J. Non-Cryst. Solids 266269, 534 (2000).

${ }^{11}$ J. Kitao, H. Harada, N. Yoshida, Y. Kasuya, M. Nishio, T. Sakamoto, T. Itoh, S. Nonomura, and S. Nitta, Solar Energy Mater. Solar Cells 66, 245 (2001).

${ }^{12}$ L. Houben, M. Luysberg, P. Hapke, R. Carius, F. Finger, and H. Wagner, Philos. Mag. A 77, 1447 (1998).

${ }^{13}$ F. Finger, J. Müller, C. Malten, and H. Wagner, Philos. Mag. B 77, 805 (1998).

${ }^{14}$ K. H. Jun, R. Carius, and H. Stiebig, Phys. Rev. B 66, 115301 (2002).

${ }^{15}$ M. Stutzmann and D. K. Biegelsen, in Amorphous Silicon and Related Materials, edited by H. Fritzsche (World Scientific, Singapore, 1988), p. 557.

${ }^{16}$ J. Müller, F. Finger, R. Carius, and H. Wagner, Phys. Rev. B 60, 11666 (1999).

${ }^{17}$ T. Dylla, Electron Spin Resonance and Transient Photocurrent Measurements on Microcrystalline Silicon, in Schriften des Forschungszentrums Jülich, Energietechnik, Vol. 43 (Forschungszentrum Jülich GmbH, Jülich, 2005).

${ }^{18}$ K. Lips, P. Kanschat, and W. Fuhs, Solar Energy Mater. Solar Cells 78, 513 (2003)

${ }^{19}$ M. Kondo, S. Yamasaki, and A. Matsuda, J. Non-Cryst. Solids 266-269, 544 (2000)

${ }^{20}$ M.M. de Lima, P.C. Taylor, S. Morrison, A. LeGeune, and F.C. Marques, Phys. Rev. B 65, 235324 (2002).

${ }^{21}$ S. Klein, F. Finger, R. Carius, and M. Stutzmann, J. Appl. Phys. 98, 024905 (2005).

${ }^{22}$ S. Klein, Microcystalline Silicon Prepared by Hot Wire CVD, in Selected Topics of Semiconductor Physics and Technology, Vol. 60, eds. G. Abstreiter, M.-C. Aman, M. Stutzmann, and P. Vogl (Verein zur Förderung des Walter Schottky Instituts der Technischen Universität München e. V, Garching, 2004)

${ }^{23}$ C. Malten, Pulsed Electron Spin Resonance of Amorphous and Microcrystalline Semiconductors, in Berichte des Forschungszentrums Jülich, Vol. 3273 (Forschungszentrum Jülich GmbH, Jülich, 1996).

${ }^{24}$ D. Ritter and K. Weiser, Opt. Commun. 57, 336 (1986).

${ }^{25}$ F. Becker, R. Carius, J.-Th. Zettler, J. Klomfaß, C. Walker, and H. Wagner, Mater. Sci. Forum 173-174, 177 (1995).

${ }^{26}$ F. Diehl, B. Schröder, and H. Oechsner, J. Appl. Phys. 84, 3416 (1998).

${ }^{27}$ F. Finger, R. Carius, T. Dylla, S. Klein, S. Okur, and M. Günes, IEE Proc.: Circuits Devices Syst. 150, 300 (2003).

${ }^{28}$ S. Klein, F. Finger, R. Carius, H. Wagner, and M. Stutzmann, Thin Solid Films 395, 305 (2001).

${ }^{29}$ S. Klein, J. Wolff, F. Finger, R. Carius, H. Wagner, and M. Stutzmann, Jpn. J. Appl. Phys., Part 2 41, L10 (2002).

${ }^{30}$ R. Carius, T. Merdzhanova, S. Klein, F. Finger, and J. Optoelectron, Adv. Mater. 7, 121 (2005) 